\title{
Uso en horticultura de los desechos orgánicos de la planta de tratamiento de Villanueva, Casanare, Colombia
}

Horticultural use of organic waste treatment plant of Villanueva, Casanare, Colombia

Leguizamo Salamanca Mery Yorleny ${ }^{1}$ y Álvarez Cohecha Eudoro²

${ }^{1}$ I. A. Universidad de los Llanos y ${ }^{2}$ I. A. Esp Docente Unillanos

\section{ealvarez@unillanos.edu.co}

Recibido 12 de Diciembre 2013, Aceptado 12 de Septiembre 2014

\section{RESUMEN}

El presente trabajo tuvo como finalidad, identificar el uso del lixiviado proveniente de la planta de tratamiento de residuos sólidos del municipio de Villanueva Casanare, y así conocer el potencial que ofrece para ser empleado como fertilizante en cultivos. En la planta de tratamiento integral de residuos sólidos en el municipio de Villanueva Casanare, el material orgánico, se muele y luego es utilizado en la lombricultura como alimento, formando un lombricompuesto, de donde posteriormente se extrajo el lixiviado para realizar un estudio sobre su potencial uso como fertilizante en cultivos de pepino cohombro (Cucumis sativus), con el fin de favorecer el desarrollo de la agricultura en la región, realizando la comparación de costos de los diferentes tratamientos empleados, para establecer la ventaja comparativa de los mismos, relacionando la presencia de insectos plagas, organismos benéficos y enfermedades en el cultivo. Los tratamientos fueron los siguientes: T0: Fertilización química, T1: 100\% de lombricompuesto, T2:100\% de lixiviado y T3: $50 \%$ de lixiviado y 50\% lombricompuesto. El cultivo de pepino cohombro respondió bien a la fertilización orgánica, sin lograr superar el testigo químico en cuanto a producción. Se obtuvo mejor rendimiento en el tratamiento químico, con un total de $3.837 \mathrm{~kg} / \mathrm{ha}$, con una rentabilidad del $14.03 \%$, mientras que con los tratamientos orgánicos, los mejores resultados se lograron en el tratamiento $50 \%$ lixiviado y $50 \%$ lombricompuesto, con un rendimiento de $3360.26 \mathrm{~kg} / \mathrm{ha}$, siendo este el tratamiento económicamente más rentable (48.1\%). 
Palabras clave: Fertilizante orgánico, lixiviado, horticultura, lombricultura.

\begin{abstract}
This study aimed to identify usage from leachate treatment plant solid waste in the municipality of Villanueva Casanare, and get to know the potential it offers for use as fertilizer on crops. On the ground of comprehensive solid waste management in the municipality of Villanueva Casanare, organic material, ground and is then used in vermiculture as food formed a earthworm-compound, where later the leachate was extracted to perform a study on the potential use as fertilizer on cucumber crops (Cucumis sativus), in order to promote the development of agriculture in the region, making the cost comparison of the different treatments used to establish the comparative advantage of them, relating the presence of insect pests, beneficial organisms and diseases. The treatments were: T0: Chemical fertilizer, T1: 100\% earthworm-compound, T2: 100\% leachate, and T3: 50\% leachate and $50 \%$ leachate earthworm-compound. Cucumber crop responded well to organic fertilization, unable to overcome the chemical control in production. Better performance in the chemical treatment, a total of $3837 \mathrm{~kg} / \mathrm{ha}$, with a yield of $14.03 \%$ was obtained while the organic treatments, the best results were achieved in $50 \%$ leachate treatment and $50 \%$ vermicompost, yield of $3360.26 \mathrm{~kg} / \mathrm{ha}$, which is the more profitable (48.1\%) treatment.
\end{abstract}

Keywords: Organic fertilizer, leachate, horticulture, vermiculture.

\title{
INTRODUCCIÓN
}

En Colombia, empresas prestadoras de servicio de aseo de ciudades capitales, donde se utilizan rellenos sanitarios mecanizados, han gastado una gran cantidad de recursos con resultados no acordes con las inversiones hechas, así mismo, los rellenos sanitarios manuales, aunque son una alternativa viable para pequeños municipios, adolecen de una alternativa para el tratamiento de lixiviados, que sea acorde con las características económicas de pequeños municipios (Pineda, 1998). 
Los lixiviados, pueden definirse como líquidos que al percolarse por las capas del suelo $u$ otro material sólido permeable, van disolviéndolo en su totalidad o a algunos de sus componentes, pueden presentar un movimiento horizontal, o sea que se desplazarán a lo largo del terreno, contaminando y dañando así el suelo y vegetación tanto del terreno como de zonas aledañas. También puede ocurrir un movimiento vertical, que penetre el subsuelo y en muchas ocasiones, alcance los mantos freáticos y acuíferos, lo que causa gigantescos problemas de contaminación del agua subterránea, principal fuente de abastecimiento de agua potable en las ciudades. Los lixiviados tienen un $\mathrm{pH}$ de 9 y presencia de una gran cantidad de sales, lo que se refleja en una alta conductividad, en ausencia de oxígeno y en alto contenido de metales pesados, como el cadmio, cromo, cobre, hierro, plomo y zinc, en concentraciones rebasan los límites de toxicidad (Ríos, 2006).

Como se mencionó, si el relleno sanitario no tiene sistema de recogida de lixiviados, éstos pueden alcanzar las aguas subterráneas y causar, como resultado, problemas medioambientales o de salud (Orta de Velásquez et al., 2006). Los peligros de estos componentes, se deben a altas concentraciones de contaminantes orgánicos y nitrógeno amoniacal, los cuales son propicios para el cultivo de microorganismos patógenos y acumulación de sustancias tóxicas (Morales, 2007).

La materia orgánica, si bien su aplicación en agricultura es milenaria, sufrió a mediados del siglo XX un olvido, causado probablemente por la introducción de los abonos químicos que producían mayores cosechas con un menor costo. No obstante, durante los últimos años se ha observado un creciente interés sobre la materia orgánica, habiendo experimentado su mercado un gran auge ligado al tema de los residuos orgánicos que encuentran así una aplicación, y al desarrollo de nuevas tecnologías (extractivas, peletización) que permiten disponer de productos comerciales de calidad. Entre los ámbitos de especial interés en los que el uso de materia orgánica es primordial, están, la agricultura sin laboreo, orgánica o biológica, y cultivo en estratos (Labrador, 2002). 
Se denomina indistintamente materia orgánica o humus a la parte que cumple un papel esencial en el suelo, no existe una definición de humus con la que todos los especialistas estén de acuerdo, pero en general, el término humus designa sustancias orgánicas variadas, de color pardo y negruzco, que resultan de la descomposición de materias orgánicas de origen exclusivamente vegetal (Julca et al., 2006). El humus tiene efecto sobre las propiedades físicas del suelo, formando agregados complejos y dando estabilidad estructural, uniéndose a las arcillas, favoreciendo la penetración del agua y su retención, y el intercambio gaseoso, disminuyendo así la erosión (Hernani, 2013). Cuando se refiere al efecto sobre las propiedades químicas del suelo, aumenta la capacidad de cambio, la reserva de nutrientes para la vida vegetal y su característica tampón favorece la acción de los abonos minerales y facilita su absorción a través de la membrana celular de las raicillas (Vargas, 2011), respecto a su efecto sobre las propiedades biológicas, afecta positivamente los procesos de mineralización, el desarrollo de la cubierta vegetal, generando nutrientes a una multitud de microorganismos que estimulan el crecimiento de la planta en un sistema ecológico equilibrado.

La cantidad de humus en el suelo depende de varios factores: incorporación de nuevos restos orgánicos y su velocidad de oxidación química, biológica, y velocidad de descomposición de la materia orgánica, la textura del suelo, la aireación, humedad y factores climáticos (López, 2009), además las prácticas de manejo del cultivo también pueden tener un efecto sobre este parámetro, ya que, por ejemplo, el empleo de abonos minerales acelera la descomposición de la materia orgánica en el suelo, lo que es una manifestación del crecimiento de la actividad biológica, que se traduce en la práctica en una mejora de la fertilidad y por tanto, de los rendimientos (Julca et al., 2006). Numerosos investigadores han reconocido efectos beneficiosos en la aplicación de la materia orgánica en el suelo, en cuanto a las mejoras observadas con respecto a las características químicas, físicas y biológicas del mismo, además ésta forma parte del ciclo del nitrógeno, del azufre y del fosforo, contribuyendo con la asimilación de nutrientes, mejorando la estructura y la retención de agua y dando soporte a todo un mundo 
de microorganismos, resultando beneficioso para el cultivo (Castellanos y Valecillos, 2011).

La materia orgánica fresca (es decir, sin descomponer) está formada por los componentes de los animales o vegetales: hidratos de carbono simples (monosacáridos) y complejos (celulosa, almidón, glucógeno, glicosilaminas, hemicelulosas y otros), compuestos nitrogenados (proteínas, ácidos nucleicos vitaminas y alcaloides.), lípidos (grasas, ácidos grasos, ceras, fosfolípidos y pigmentos.), ácidos orgánicos (cítrico, fumárico, málico, malónico y succínico), polímeros y compuestos fenólicos (ligninas y taninos) y elementos minerales (Labrador, 2002).

En la composición del humus se encuentra un complejo de macromoléculas en estado coloidal constituido por proteínas, azúcares, ácidos orgánicos y minerales, en constante estado de degradación y síntesis (Pérez, 2003). El humus, por tanto, abarca un conjunto de sustancias de origen muy diverso, que desarrollan un papel de importancia en la fertilidad, conservación y presencia de vida en los suelos. A su vez, la descomposición del humus en mayor o menor grado, produce una serie de productos coloidales que, en unión con los minerales arcillosos, originan los complejos organominerales que aglutinados determinan la textura y estructura de un suelo, además los coloides existentes en el suelo presentan además carga negativa, hecho que les permite absorber cationes $\mathrm{H}^{+}$y metálicos $\mathrm{Ca}^{2+}, \mathrm{Mg}^{2+}, \mathrm{K}^{+}$, $\mathrm{Na}^{+}$haciendo intercambios en todo momento de forma reversible, debido a este hecho, los coloides también reciben el nombre de complejo absorbente (Pérez, 2013). En la Tabla 1 se presenta un resumen sobre los beneficios del humus.

Se entiende por lombricultura las diversas operaciones relacionadas con la cría y producción de lombrices y el tratamiento de residuos orgánicos para su reciclaje en forma de abonos y proteínas, es una tecnología basada en su cría intensiva para la producción de humus a partir de un sustrato orgánico, donde existe un proceso de descomposición natural, similar al compostaje, en el que el material orgánico, además de ser atacado por los microorganismos (hongos, bacterias, 
actinomicetos y levaduras) también lo es por el complejo sistema digestivo de la lombriz (Mamani et al., 2012).

Tabla 1. Influencia del humus en el suelo

\begin{tabular}{|c|c|c|}
\hline Física & Química & Biológica \\
\hline $\begin{array}{l}\text { Incrementa capacidad de } \\
\text { intercambio catiónico }\end{array}$ & Regula nutrición vegetal & $\begin{array}{c}\text { Aporta microorganismo } \\
\text { útiles }\end{array}$ \\
\hline $\begin{array}{l}\text { Da consistencia a suelos } \\
\text { ligeros y compactos }\end{array}$ & $\begin{array}{l}\text { Mejora intercambio de } \\
\text { iones }\end{array}$ & $\begin{array}{l}\text { Sirve de soporte y } \\
\text { alimento de a los } \\
\text { microorganismo }\end{array}$ \\
\hline $\begin{array}{c}\text { Facilita labranza de la } \\
\text { tierra }\end{array}$ & $\begin{array}{l}\text { Mejora la asimilación de } \\
\text { abonos minerales }\end{array}$ & $\begin{array}{c}\text { No tiene semillas } \\
\text { perjudiciales (arvenses) }\end{array}$ \\
\hline $\begin{array}{l}\text { Evita formación de } \\
\text { costras y compactación }\end{array}$ & $\begin{array}{l}\text { Mejora la disponibilidad } \\
\text { de fosforo y potasio }\end{array}$ & $\begin{array}{c}\text { Mejora la resistencia de } \\
\text { las plantas }\end{array}$ \\
\hline $\begin{array}{l}\text { Ayuda a la retención y } \\
\text { drenaje de agua e } \\
\text { Incrementa porosidad }\end{array}$ & $\begin{array}{l}\text { Produce gas carbónico } \\
\text { que mejora la solubilidad } \\
\text { de los minerales }\end{array}$ & $\begin{array}{c}\text { Mejora la reproducción } \\
\text { sexual }\end{array}$ \\
\hline
\end{tabular}

Fuente: Adaptado de Pérez, (2013).

En el intestino de la lombriz ocurren procesos de fraccionamiento, desdoblamiento, síntesis y enriquecimiento enzimático y microbiano, lo cual tiene como consecuencia un aumento significativo en la velocidad de degradación y mineralización del residuo, obteniendo un producto de alta calidad. Esta transformación hace que los niveles de pérdida de nutrientes como nitrógeno y potasio, sean mínimos con relación a los sistemas tradicionales de compostaje, resultando tres productos: el humus, lombrices y lixiviados, la lombricultura doméstica, es practicada por personas con alto sentido de la ecología para reciclar sus residuos domésticos, de cocina y jardín (Mendoza, 2008), por otra parte, la lombricultura ofrece una buena alternativa para el tratamiento de residuos orgánicos contaminantes, tales como restos de cosechas, desperdicios de restaurantes, estiércoles, residuos industriales de origen orgánico en plantas de sacrificio, papeleras, agro industrias y otros.

El humus tiene una composición de $57.64 \%$ de humedad, $70.79 \%$ de materia orgánica, $2.91 \%$ de nitrógeno, $2.01 \%$ de fósforo, $1.80 \%$ de potasio, $4.60 \%$ de 
calcio, $0.64 \%$ de magnesio, $0.60 \%$ de hierro y altas concentraciones de manganeso, cobre, zinc y cobalto. Las lombrices ingieren diariamente una cantidad de comida equivalente a su propio peso y expelen el $60 \%$ transformado en humus ( $0.3 \mathrm{~g} / \mathrm{día})$ que es un abono orgánico prácticamente insuperable, que puede incrementar hasta en un $300 \%$ la producción de hortalizas y otros productos vegetales, se pueden obtener grandes cantidades de humus en pequeñas superficies (Moya, 2011).

El compostaje es el humus obtenido de manera natural por descomposición bioquímica al favorecer la fermentación aeróbica de residuos orgánicos como restos vegetales, animales y excrementos, por medio de la reproducción masiva de bacterias aeróbicas termófilas que están presentes en forma natural en cualquier lugar, posteriormente, la fermentación la continúan otras especies de bacterias, hongos y actinomicetos (Sztern y Pravia, 1999). Normalmente, se trata de evitar la putrefacción de los residuos orgánicos por exceso de agua, que impide la aireación y oxigenación, lo cual crea condiciones biológicas anaeróbicas malolientes.

El compostaje además de su utilidad directa, implica una solución estratégica y ambientalmente aceptable a la problemática planteada por las grandes concentraciones urbanas (y sus residuos sólidos orgánicos domésticos) y las explotaciones agrícolas, forestales y ganaderas, cuyos residuos orgánicos deben ser tratados, también se puede considerar una tecnología alternativa a otras que no siempre son respetuosas con los recursos naturales y el medio ambiente y que además tienen un costo elevado (Sztern y Pravia, 1999).

El pepino cohombro, se originó en Asia tropical, su cultivo (Figura 1) exige alta temperatura y humedad relativa, sin embargo, se adapta a climas a fríos y templados y necesita suelos fértiles y bien drenados, desde los arenosos hasta los franco-arcillosos, contando con una profundidad efectiva mayor de $60 \mathrm{~cm}$ que facilite la retención del agua y el crecimiento del sistema radicular para lograr un buen desarrollo y excelentes rendimientos. En cuanto a $\mathrm{PH}$, el cultivo se adapta a un rango de 5.5-6.8. (Argona et al., 1992). 


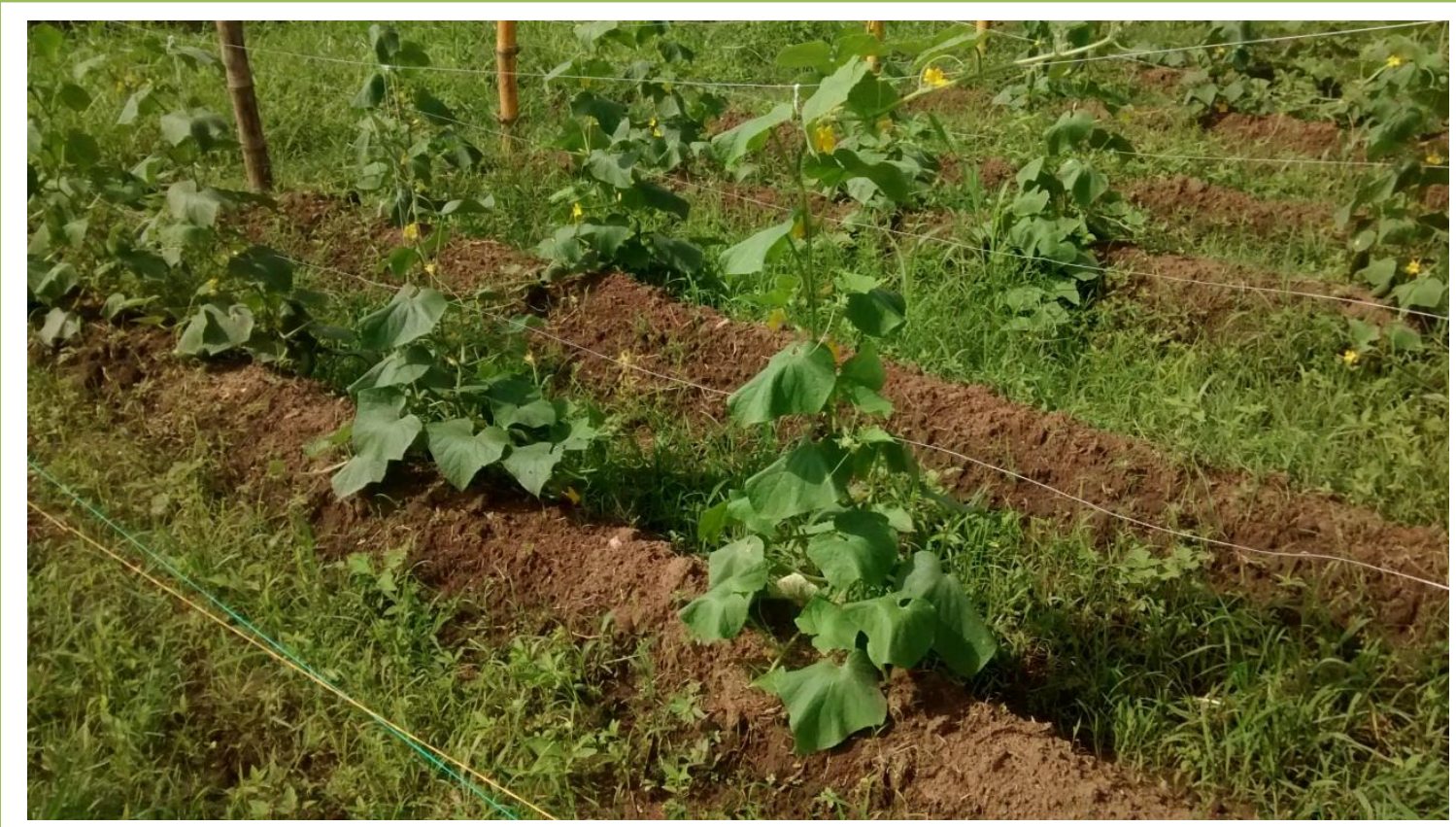

Figura 1. Cultivo de pepino en establecimiento.

Fotografía: Grupo de investigación en agroforestería

En experimentos en Colombia se han observado producciones de $7.9 \mathrm{~kg} \mathrm{~m}$ presentando una precocidad de 87 días desde su trasplante al inicio de la cosecha, y se han utilizados fertilizantes de uso más extendido los simples en forma de sólidos solubles (nitrato cálcico, nitrato potásico, nitrato amónico, fosfato monopotásico, fosfato monoamónico, sulfato potásico y sulfato magnésico) y en forma líquida (ácido fosfórico y ácido nítrico), debido a su bajo costo y a que permiten un fácil ajuste de la solución nutritiva, aunque existen en el mercado abonos complejos sólidos cristalinos que se ajustan adecuadamente, solos o en combinación con los abonos simples, para suplir los requerimientos en las distintas fases de desarrollo del cultivo. El uso de compostaje permite mejorar la producción y reducir la dependencia de insumos químicos que van en detrimento del medio ambiente, obteniéndose por métodos culturales y de implementación de técnicas que utilizan lixiviados de compostaje, favoreciendo la nutrición y sanidad de cultivos, a bajos costos (Larco, 2004). Los lixiviados son todos aquellos líquidos que han entrado en contacto con los desechos de rellenos sanitarios, y se degradan por la disolución de uno o más compuestos en contacto con el agua de residuos sólidos provenientes de las ciudades. 


\section{METODOLOGÍA}

El trabajo de campo se realizó, en el segundo semestre del 2010, en un terreno ubicado en la vereda Puerto Rosales, Municipio de Villanueva, ubicado al sur del departamento del Casanare, sobre la parte baja del piedemonte, $300 \mathrm{msnm}$ y una temperatura promedio $25.7^{\circ} \mathrm{C}$. Estos suelos pertenecen a la clase IV y sus características químicas se observan en los resultados del análisis de suelo (Tabla 2). Para la elección del terreno se tuvo en cuenta que este fuera lo más homogéneo posible y cerca de un lugar donde se pudiera tomar agua para riego en caso que se necesitara, la toma de muestra de suelos se hizo teniendo en cuenta todos los aspectos que se recomiendan para obtener resultados representativos y confiables, la preparación del terreno en su totalidad se hizo en forma manual.

Tabla 2. Análisis químico de suelos del sitio donde se realizó la evaluación

\begin{tabular}{ccccccccccccccc}
\hline Textura & $\mathbf{M} . \mathbf{0}$ & $\mathbf{P}$ & $\mathbf{p H}$ & \multicolumn{11}{c}{ Cationes meq/100 g suelo } \\
\hline Franco & $\%$ & ppm & & $\mathrm{Al}$ & $\mathrm{Ca}$ & $\mathrm{Mg}$ & $\mathrm{K}$ & $\mathrm{Na}$ & $\mathrm{B}$ & $\mathrm{Fe}$ & $\mathrm{Cu}$ & $\mathrm{Mn}$ & $\mathrm{Zn}$ & $\mathrm{S}$ \\
Arcilloso & 0.9 & 27 & 4.1 & 0.85 & 0.7 & 0.25 & 0.08 & 0.01 & 0.25 & 413 & 0.7 & 5.6 & 2 & 1 \\
\hline
\end{tabular}

Fuente: Laboratorio de suelos UNILLANOS

Se utilizó un diseño experimental de bloques completos al azar (4 tratamientos, 3 repeticiones), para un total de 12 parcelas de $12 \mathrm{~m}^{2}$ cada una, el análisis de los datos se realizó en el software estadístico SAS ver 9.0, las variables evaluadas se agruparon en dos campos: el componente rendimiento de acuerdo con el tipo de fuente nutricional y la relación e interacción del lixiviado con la presencia de insectos plagas, benéficos y enfermedades en el cultivo.

Se trabajó con 3 bloques o repeticiones de $16 \mathrm{~m}$ de largo por $3 \mathrm{~m}$ de ancho para un área de $48 \mathrm{~m}^{2}$ por bloque, cada uno estuvo compuesto por 4 parcelas de 12 $\mathrm{m}^{2}$, la distancia o separación entre cada uno de los bloques, parcelas y borde fue 1 metro, siendo el área efectiva del experimento fue $273 \mathrm{~m}^{2}$. Se sembraron tres semillas por sitio, debidamente tratadas con Trichoderma sp., a una profundidad de $2 \mathrm{~cm}$, para ralear 2 y dejar una planta definitiva, a una distancia entre sí de 40 
cm y un $1 \mathrm{~m}$ entre surcos, para el tutorado se utilizó un sistema de apoyo de espaldera simple con una sola línea a 2,5 m de altura

Para la evaluación del uso del lixiviado como nutriente en el cultivo de pepino cohombro, se evaluó el desarrollo y producción del cultivo con fertilización química, con tres alternativas orgánicas, para un total de 4 tratamientos con tres repeticiones cada uno, se sembró en camas, a $40 \mathrm{~cm}$ entre plantas y $1 \mathrm{~m}$ entre surco para un total de 18 plantas por cama. Los tratamientos en cada bloque fueron asignados al azar, su descripción y fertilización se observan en la Tabla 3. El trazado de las parcelas se hizo con ayuda de decámetro, estacas y pita, se aplicó $0.87 \mathrm{~kg}$ de cal dolomita por parcela y la fertilización que se muestra en la Tabla 4.

Para evaluar el componente rendimiento, se efectuaron 5 muestreos en donde se tomó el número total de frutos producidos en el cultivo, separándolos por tratamiento, luego se pesaron y midieron, y con estos datos se realizó una comparación entre medias utilizando la prueba de Tukey.

Tabla 3. Descripción de tratamientos y cantidad fertilizante aplicado en cada tratamiento

\begin{tabular}{ccc}
\hline Tratamiento & Descripción \\
\hline T0 & Fertilización química & $\begin{array}{c}210 \mathrm{~g} \text { triple } 18,175 \mathrm{~g} \text { sulfak, } 55 \mathrm{~g} \\
\text { elementos menores por parcela. }\end{array}$ \\
T1 & $\begin{array}{c}\text { Orgánico: } \\
\text { 100\% Lombricompuesto } \\
\text { Orgánico: }\end{array}$ & $100 \mathrm{~g}$ por parcela. \\
T2 & $\begin{array}{c}\text { 100\% Lixiviado } \\
\text { Orgánico: }\end{array}$ & $30 \mathrm{ml}$ por aplicación por planta \\
50\% Lixiviado, & $\begin{array}{c}\text { Lixiviado (30 ml/aplicación/planta), } \\
\text { T3 }\end{array}$ & Lombricompuesto $100 \mathrm{~g}$ por parcela \\
\hline
\end{tabular}

El control de malezas se realizó en forma manual con pala, a los 10, 25 y 40 días después de la siembra, el problema más grave de plagas que se presentó fue Diaphania sp., también se presentó ataques de áfidos y otras plagas, pero sin ocasionar daño económico. La aparición de enfermedades fue esporádica sin presentar mayor daño, por lo que se tomó la decisión de no hacer aplicaciones de 
agroquímicos, viéndose ataques de fumagina (Capnodium sp.) especialmente. Se realizaron 5 recolecciones, tomando la totalidad de los frutos.

Tabla 4. Aplicación de la fertilización en cada tratamiento

\begin{tabular}{|c|c|}
\hline Tratamiento & Descripción \\
\hline T0 & $\begin{array}{l}\text { El } 50 \% \text { al momento de la siembra (AMS), 50\% } 20 \text { días antes de } \\
\text { la floración. }\end{array}$ \\
\hline T1 & $100 \%$ al momento de la siembra (AMS). \\
\hline T2 & $\begin{array}{l}\text { Primera aplicación al momento de la siembra (AMS), segunda } \\
\text { aplicación } 8 \text { días después de la germinación (DDG), tercera } \\
\text { aplicación } 10 \text { días antes de la floración. }\end{array}$ \\
\hline T3 & $\begin{array}{l}\text { Lombricompuesto ( } 100 \% \text { al momento de la siembra (AMS)) } \\
\text { Lixiviado: primera aplicación al momento de la siembra (AMS), } \\
\text { segunda aplicación } 8 \text { días después de la germinación (DDG), } \\
\text { tercera aplicación } 10 \text { días antes de la floración. }\end{array}$ \\
\hline
\end{tabular}

\section{RESULTADOS Y DISCUSIÓN}

En cuanto a tamaños y pesos de los frutos (Figura 2) se ve claramente que el tamaño de los frutos en los 4 tratamientos fue uniforme, contrario al peso en el que los mejores resultados se obtuvieron en el testigo (T0), seguido del tratamiento químico, se logró comprobar que el T3 es una muy buena alternativa como abono, para el manejo del cultivo de pepino, ya que la diferencia de pesos con respecto al T0 es $60 \mathrm{~g}$, por lo que se puede deducir que económicamente es viable la producción orgánica, además se obtiene un producto inocuo de excelente calidad para el mercado.

Aunque en el tratamiento T2 se presentaron los más bajos pesos de los frutos (400 g), se comprobó que el lixiviado si es una buena fuente nutricional en la producción de pepino, además su aplicación no genera ningún problema patológico al cultivo, ya que las plantas no presentaron síntomas que pudieran alertar sobre la presencia de alguna enfermedad.

Los tratamientos $\mathrm{T} 1, \mathrm{~T} 2$ y $\mathrm{T} 3$ presentaron su pico máximo de producción aproximadamente a los 72 días, en la quinta recolección (R5), lo que indica que el 
lombricompuesto y el lixiviado ejercen una mejor función al aplicarlos antes de la siembra, contrario a lo que sucedió con el T0 que su pico máximo de producción se presentó a los 62 días después de sembrado el cultivo en la recolección 3 (R3) (Figura 3).

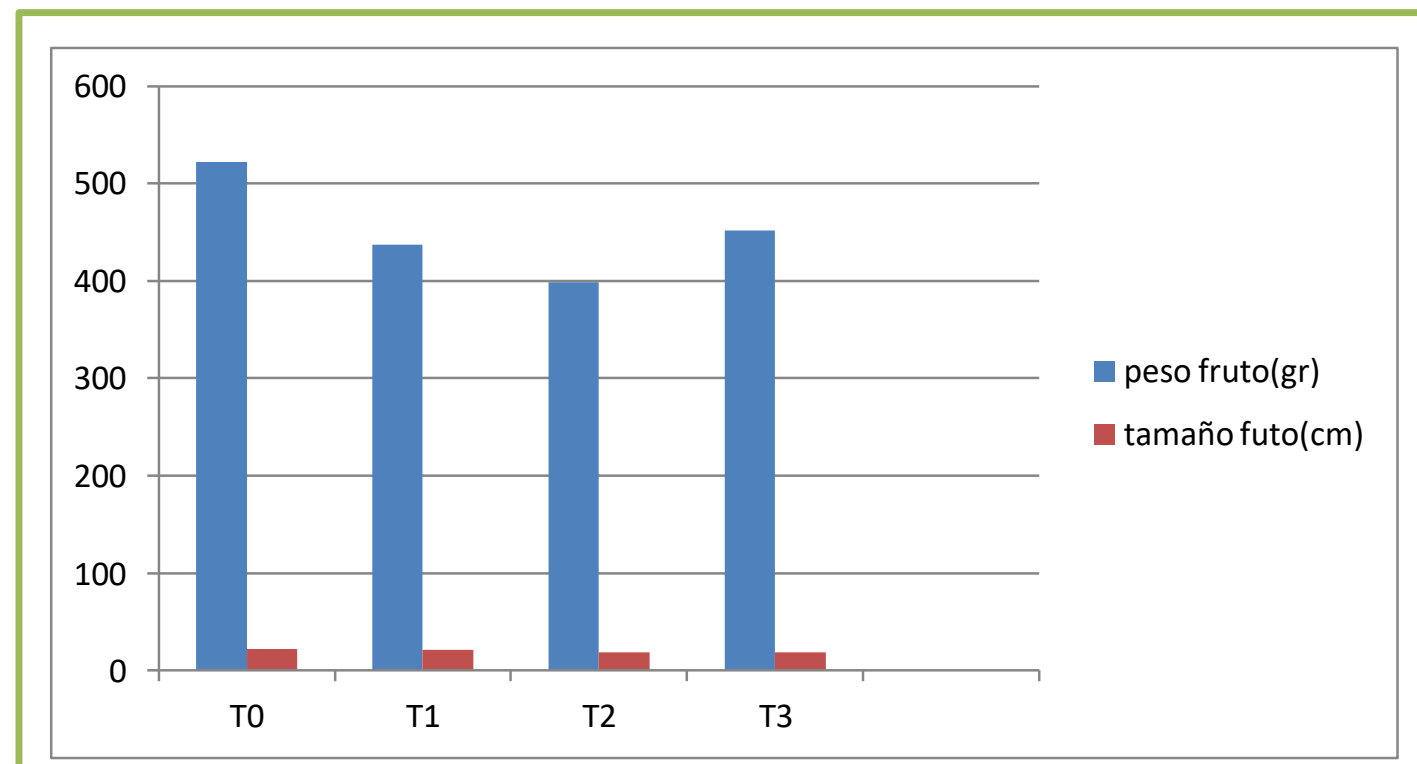

Figura 2. Peso Vs tamaño del fruto en cada tratamiento

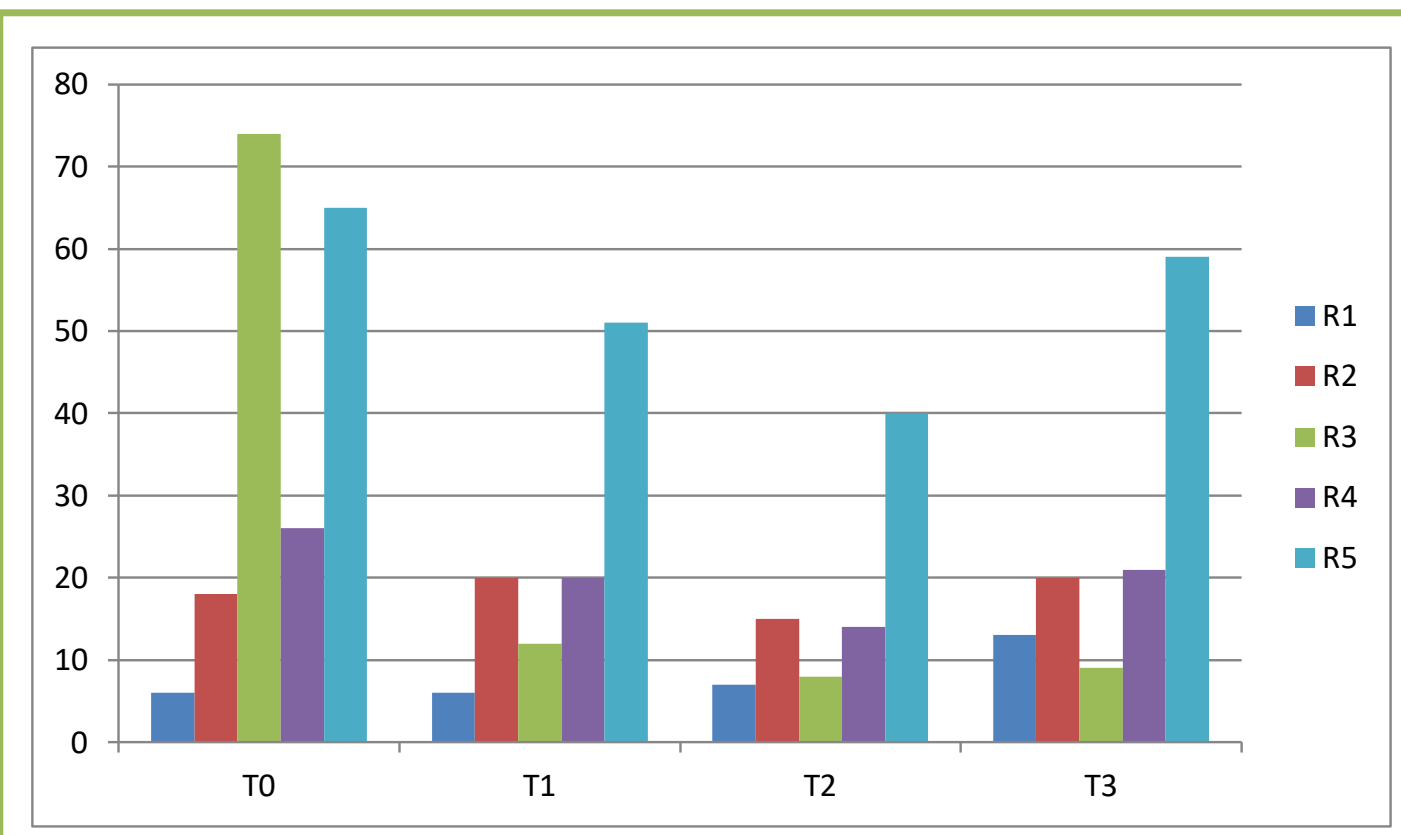

Figura 3. Tratamiento Vs número de frutos recolectados

Al realizarse un análisis entre las Figuras 1 y 2 , se puede deducir que el número de frutos no tiene mayor incidencia respecto al total de la producción, ya que el T0 
aunque presenta mayor número de frutos recolectados y mayor peso de los mismos, al compararlo con los pesos presentados por el T3, la diferencia es poca 15 frutos $120 \mathrm{~g}$, considerando que este posee menor número de frutos cosechados. Aunque en el T2, se obtuvieron los resultados más bajos en cuanto a número 40, tamaño $10 \mathrm{~cm}$ y peso $400 \mathrm{~g}$ de los frutos, se probó que es una alternativa válida como fertilizante orgánico en la producción de pepino cohombro. Según la prueba de comparación medias, prueba de Tukey, para peso de fruto (Tabla 5), T0 no presenta diferencia con T1, pero si con T2 y T3, aunque T1 fue similar $(\mathrm{P}<0.05)$ con estos últimos tratamientos.

Para tamaño de fruto (Tabla 6), T0 es semejante con T1, pero si es mayor con relación al T2 y T3, aunque el valor de T1 es similar $(\mathrm{P}<0.05)$ con los de T0 y T2, siendo menor el tamaño en T3, sin embargo, es importante aclarar que el rango de diferencia significa es bajo $(1.88 \mathrm{~cm})$, por lo que no puede ser tan influyente en el momento de la comercialización.

Tabla 5. Prueba de comparación medias, prueba de Tukey, para peso de fruto Diferencia significativa mínima 93.504

Tukey Agrupamiento

A

B A

B

B
Media

526.34

437.55

430.11

394.68

N

14

15

15

13
Tratamiento

T0. Químico

T1. Lombricultivo

T3. $50 \%$ Lixiviado

T2. Lixiviado

Medias con la misma letra no son significativamente diferentes.

Tabla 6. Prueba de comparación medias, prueba de Tukey, para tamaño de fruto

Diferencia significativa mínima

\begin{tabular}{cccl} 
Tukey Agrupamiento & Media & N & \multicolumn{1}{c}{ Tratamiento } \\
\hline A & 22.1571 & 14 & T0. Químico \\
B A & 20.8467 & 15 & T1. Lombricultivo \\
B C & 19.6067 & 15 & T3. 50\% Lixiviado \\
C & 18.4385 & 13 & T2. Lixiviado \\
\hline
\end{tabular}


Teniendo en cuenta los resultados en el análisis económico, se encontró que según la cantidad de producto vendido, los mayores ingresos por ventas por hectárea, se obtuvieron con el tratamiento (T0) $\$ 1.918 .958,33$, seguido del tratamiento (T1) $\$ 1.709 .166,67$, luego el tratamiento (T3) $\$ 1.680 .130,21$ y por último el tratamiento (T2) $\$ 1.336 .145,83$, en el cual se obtuvo la menor producción y por ende los menores ingresos por ventas.

Respecto a costos de producción por hectárea en estos se presentó la misma secuencia de las ventas, más elevados para T0: $\$ 1.682 .750,00$ seguido T1: $\$ 1.217 .700,00$, luego T3: $\$ 1.134 .500,00$ y por último T2: $\$ 1.041 .300,00$, en el cual se obtuvo los menores costos, cuya diferencia se dio por los fertilizantes utilizados en cada tratamiento. Al realizar la comparación de los costos vs los ingresos por ventas, se observa que el tratamiento que presenta una mayor utilidad, es el T3: $\$ 545.630,00$, seguido de T1: $\$ 491.466,00$, luego T2: $\$ 294.845,00$ y por último T0: $\$ 236.208,00$, esto debido a que en este tratamiento se tienen los mayores costos de producción, teniendo en cuenta que es el tratamiento testigo o tratamiento químico (Tabla 7).

Al llevar estos resultados a un porcentaje de rentabilidad, se obtuvo la mayor rentabilidad con T3: $48.1 \%$, seguido por T1: $40.36 \%$, luego T2: $28.31 \%$ y por último T0: $14.03 \%$, en estos casos es importante resaltar que, aunque en T2 se presentan los menores ingresos por ventas, este tiene una mayor rentabilidad que el T0 el cual presenta los mejores ingresos por ventas.

Tabla 7. Comparación utilidad obtenida en los 4 tratamientos

\begin{tabular}{cccc}
\hline Tratamiento & $\begin{array}{c}\text { Costo de } \\
\text { producción }\end{array}$ & Ventas & Utilidad \\
\hline T0. Trat Quím & $\$ 1.682 .750,00$ & $\$ 1.918 .958,00$ & $\$ 236.208,00$ \\
T1. Lombric & $\$ 1.217 .700,00$ & $\$ 1.709 .166,00$ & $\$ 491.466,00$ \\
T2. Lixiv & $\$ 1.041 .300,00$ & $\$ 1.336 .145,00$ & $\$ 294.845,00$ \\
T3. 50\% Lixiv & $\$ 1.134 .500,00$ & $\$ 1.680 .130,00$ & $\$ 545.630,00$ \\
\hline
\end{tabular}

En términos generales el cultivo presentó un buen desarrollo, el inicio de la floración se dio a los 29-30 días después de sembrado y la primera cosecha se 
hizo a los 52 días, alcanzando un tope máximo de producción al final de la cosecha para los tratamientos orgánicos y a los 62 días después de la siembra para el tratamiento químico, lo que permite deducir que los tratamientos orgánicos no afectaron el ciclo normal de producción del cultivo

Es importante anotar que los ataques de plagas y enfermedades, presentes en la etapa del cultivo, no tuvieron mayor influencia en su ciclo, ni en la producción, por lo que no se realizaron aplicaciones de químicos.

\section{CONCLUSIONES}

El cultivo de pepino cohombro respondió bien a la fertilización orgánica, sin lograr superar el testigo químico en cuanto a producción que obtuvo mejor rendimiento, con un total de $3837 \mathrm{~kg} / \mathrm{ha}$, y rentabilidad del $14.03 \%$, mientras que, con los tratamientos orgánicos, los mejores resultados se lograron en el tratamiento 50\% lixiviado y $50 \%$ humus, con un rendimiento de $336026 \mathrm{Kg} / \mathrm{ha}$, siendo este el tratamiento económicamente más rentable (48.1\%).

Aunque todos los tratamientos superaron al T2 (100\% lixiviado), en cuanto a rendimiento, es importante aclarar que este, es económicamente más rentable que el tratamiento químico, obteniendo un $28.31 \%$, frente al $14.03 \%$ respectivamente, lo que lo convierte en una opción promisoria en la producción de pepino.

Frente a la utilización del $100 \%$ lixiviado como fertilizante, se obtuvo una producción de $2672.26 \mathrm{~kg} / \mathrm{ha}$, con una rentabilidad del $28.31 \%$, mientras que al combinar éste con el humus, se aumenta la producción en $688 \mathrm{~kg} / \mathrm{ha}$ y su rentabilidad en un $19.79 \%$, lo cual indica que el lixiviado es más efectivo en la producción, al utilizarlo como complemento del humus.

Los picos máximos de producción para los tratamientos orgánicos (T1, T2, T3), se obtuvieron aproximadamente a los 72 días después de sembrado el cultivo, contrario a lo que sucedió con el tratamiento químico (T0), que su pico máximo de producción se presentó a los 62 días después de sembrado el cultivo, lo que indica que la fertilización tuvo influencia en el ciclo del cultivo. 


\section{RECOMENDACIONES}

Promover el uso de abonos orgánicos en las personas dedicadas al cultivo de pepino, con el fin de mejorar las relaciones con el medio ambiente y a la vez disminuir costos de producción, lo cual es benéfico para todos.

Establecer la composición físico-química y microbiológica del lixiviado, en el laboratorio, para que de esta manera, se pueda dar un mejor uso de este, aplicando dosis apropiadas para la producción, en el cultivo que se desee emplear.

Aconsejar a los productores de pepino, el uso de él lixiviado en complemento con el humus, como una alternativa orgánica en el manejo de este cultivo.

Realizar investigaciones semejantes, utilizando diferentes dosis de lixiviado solo y asociado con el humus, en busca de un mayor aporte de nutrientes que mejore la producción y a la vez la rentabilidad del cultivo.

Ejecutar una investigación, aplicando el lixiviado como fertilizante foliar y en otros cultivos, para que de esta manera se logre ampliar su rango de utilización en la agricultura.

\section{BIBLIOGRAFÍA}

1. Castellanos F., Valecillos M. Diseño de módulo de lombricultura. Servicio y soporte para la elaboración de Biofertilizante sólido. Universidad de los Andes, Núcleo Universitario Rafael Rangel, Departamento de ciencias Agrarias. INSAI - Pampanito, Estado Trujillo. Tesis de grado para optar al título de Técnico Superior Agrícola, 61 p. 2011.

2. Finck, A Fertilizantes y fertilización. Fundamentos y métodos para la fertilización de los cultivos. Editorial Reverte, Barcelona, España, p 157-171. 1988.

3. Green J., Flores L., Sánchez V. Inteligencia del mercado del pepino. Ed Centro de Investigaciones Biológicas del Noreste, S.C. La Paz, Baja California del Sur, México, 85 p. 2012.

4. Hernani N. Comportamiento agronómico de dos variedades de frutilla (Fragaria sp.) con la aplicación de dos niveles de humus de lombríz y el bio-fertilizante (zumia-15) en ambiente protegido - Cota - La Paz. Tesis de Pregrado para optar al título de Ingeniero Agrónomo. Facultad de Agronomía, Universidad Mayor de San Andrés, Bolivia. 127 p. 2013.

5. Julca O., Meneses F., Blas S., Bello A. La materia orgánica, importancia y experiencia de su uso en la agricultura. Revista Idesia (Arica), 24 (1): 49-61. 2006.

6. Labrador M. La materia orgánica en los agrosistemas: aproximación al conocimiento de la dinámica, la gestión y la reutilización de la materia orgánica en los agrosistemas. Ed. Ministerio de Agricultura, Pesca y Alimentación, España. 293 p. 2002. 
7. Larco E. Desarrollo y evaluación de lixiviados de compost y lombricompost para el manejo de Sigatoka Negra (Mycosphaerella fijiensis Morelet) en plátano. Tesis de grado para optar al título Magister Scientiae. Centro Agronómico Tropical de Investigación y Enseñanza CATIE. Programa de educación para el desarrollo y la conservación, Escuela de posgrados, Turrialba, Costa Rica, 77 p. 2004.

8. López A. Las reservas orgánicas edáficas y su relación con la capacidad de almacenamiento de agua de los suelos agrícolas. Tesis de grado para optar al título de Maestro en ciencias. Colegio de Posgraduados, Montecillo, Texcoco, México, 104 p. 2009.

9. López E., Rodríguez J., Huez M.., Garza S., Jiménez J., Leyva E. Producción y calidad de pepino (Cucumis sativus L.) bajo condiciones de invernadero usando dos sistemas de poda. Revista electrónica Idesia (Arica), 29 (2): 21-27. 2011.

10. Mamani G., Mamani P., Sainz H., Vilca R. Comportamiento de la lombriz roja (Eisenia spp.) en sistemas de vermicompostaje de residuos orgánicos. Journal of the Selva Andina Research Society. 3 (1): 44-54. 2012.

11. Mendoza L. Manual de lombricultura. SEP, Tuxtla Gutiérrez, México. 39 p. 2008. Disponible En: http://www.cecytech.edu.mx/Pdf/manuallombricultura.pdf

12. Morales. J. Estudio para la remoción de metales pesados en los lixiviados de rellenos sanitarios. Trabajo de grado para optar al título de especialista en Ingeniería Ambiental con énfasis en Ingeniería Sanitaria. Facultad de Ingeniería y Arquitectura, Universidad Nacional de Colombia, Manizales, 96 p. 2007.

13. Moya J. Estudio de la calidad nutricional de la vermiharina de lombriz (Eisenia foetida) liofilizada como materia prima para la elaboración de balanceado para alevines de trucha arco iris (Oncorhynchus mykiss). Tesis de grado para optar al título de Bioquímico Farmacéutico. Facultad de ciencias, Escuela de Bioquímica y Farmacia. Escuela Superior Politécnica de Chimborazo, Riobamba, Ecuador, 99 p. 2011

14. Sztern D., Pravia M. Manual para la elaboración del compost, Bases conceptuales y conceptos. Organización Panamericana de la Salud (OPS). Oficina de planeamiento y presupuesto, Unidad de desarrollo municipal. 67 p. 1999.

15. Orta de Velásquez M., Rojas M., Yañez I., Mongue I., Londoño J. Alternativa de tratamiento de lixivados de rellenos sanitarios en plantas de aguas residuales urbanas. Revista AIDIS de Ingeniería y Ciencias Ambientales, 1 (1). 2006. Disponible En: http://www.revistas.unam.mx/index.php/aidis/article/view/14445/13780

16. Pérez E. Análisis de fertilidad de suelos en el laboratorio de Química del Recinto de Grecia, Sede de Occidente, Universidad de Costa Rica, Revista Intersedes: Revista de las Sedes Regionales. XIV (29): 6-18. 2013.

17. Pérez J. Cinética de la lombriz de tierra Eisenia fétida (Edwards y Bholen, 1996) en la generación de Humus para la producción de Nopal Verdura. Tesis de grado para optar al título de Maestro en ciencias en producción agrícola. Universidad Autónoma de Nuevo León. Facultad de Agronomía, 104 p. 2003.

18. Pineda, S. Manejo y disposición de residuos sólidos urbanos. Ed. Asociación Colombiana de Ingeniería Sanitaria y Ambiental. Bogotá, 388 p. 1998.

19. Ríos S. Estudio de un método fisicoquímico alternativo para la remoción de carga orgánica del lixiviado proveniente del relleno sanitario "El Carrasco". Tesis de pregrado para optar al Título de Químico. Facultad de ciencias, Escuela de Química. Universidad Industrial de Santander, Bucaramanga, 130 p. 2006.

20. Vargas V. Evaluación de diferentes dosis de enmiendas húmicas en la producción primaria de forraje del Lolium perenne (Rye Grass). Tesis de Grado. Escuela Superior Politécnica de Chimborazo. Facultad de Ciencias Pecuarias. Escuela de Ingeniería Zootécnica. Riobamba - Ecuador. 21 p. 2011. 